\section{Acute endophthalmitis after cataract surgery at a referral centre in Northern Taiwan: review of the causative organisms, antibiotic susceptibility, and clinical features}

J-H Cheng, Y-H Chang, C-L Chen, Y-H Chen, D-W Lu and J-T Chen

\begin{abstract}
Purposes The aims of this study were to describe the bacterial isolates and treatment outcomes of endophthalmitis after cataract surgery at a referral centre in Northern Taiwan. Methods This was a retrospective, interventional, and observational series. Medical records were review of patients with clinical diagnosed, and/or culture-proven bacterial endophthalmitis at a tertiary referral centre in Northern Taiwan from January 2002 to December 2008. Treatment generally conformed to standard guidelines. Main outcome measurements were results of organism culture, antibiotic susceptibility of isolated bacteria, and visual acuity (VA) outcome.

Results Thirty-four patients had positive cultures. A total of $55.9 \%$ of the culturepositive isolates were Gram negative and 44.1\% were Gram positive. Pseudomonas aeruginosa was the most commonly isolated organism. Eighteen patients had final VA better than 5/200, and 41 had final VA worse than $5 / 200$. In multiple linear regression analysis, female gender, presence of hypopyon, and worse baseline VA were significant independent predictors of worse visual outcome.

Conclusions It was found that at a referral centre in Northern Taiwan, the majority of bacterial isolates were Gram negative in acute

postoperative bacterial endophthalmitis after cataract surgery and $P$. aeruginos $a$ was the most common bacterial isolate.

Eye (2010) 24, 1359-1365; doi:10.1038/eye.2010.39; published online 9 April 2010

Keywords: acute endophthalmitis; cataract surgery; pars plana vitrectomy; intravitreal antibiotics; Pseudomonas aeruginosa;

Staphylococcus aureus

\section{Introduction}

Cataract extraction is a very commonly preformed ophthalmic surgical procedure. An uncommon but serious complication of this procedure is postoperative bacterial endophthalmitis. ${ }^{1}$ In a US Medicare study, the incidence of bacterial endophthalmitis after cataract surgery was reported to be 2.15 per $1000 .^{2}$ Studies in Canada, Scotland, South India, and Saudi Arabia have found the incidence to be $0.043,0.2,0.05$, and $0.068 \%$, respectively. ${ }^{3-6}$ This complication requires urgent identification of the causative organism by culturing intraocular aspirates and treatment with effective antibiotics to prevent a poor visual outcome. Therapeutic strategies are modified according to the results of culture and the clinical features. However, before culture results are available, empiric treatments based on the epidemiological findings in the community may be used.
\end{abstract}

Department of

Ophthalmology, Tri-Service General Hospital, National Defense Medical Center, Taipei, Taiwan, Republic of China

Correspondence: J-T Chen, Department of Ophthalmology, Tri-Service General Hospital, National Defense Medical Center,

No. 325, Section 2, Cheng-Kung Road, Taipei 114, Taiwan, Republic of China Tel: + 886287923100 12529;

Fax: + 886287927164 .

E-mail: jt66chen@ ms32.hinet.net

Received: 8 October 2009 Accepted in revised form: 26 February 2010 Published online: 9 April 2010 
There have been a number of studies carried out to identify the causative organisms of postoperative bacterial endophthalmitis. The Endophthalmitis Vitrectomy Study ${ }^{7}$ (EVS) and geographic surveys carried out in Sweden, ${ }^{8}$ Singapore, ${ }^{9}$ southwest Finland, ${ }^{10}$ and the United Kingdom ${ }^{11}$ reported that the majority of cases of postoperative bacterial endophthalmitis were caused by Gram-positive organisms, which are normal flora of the external eyes. However, it has been noted that over time, there have been changes in the microbiologic spectrum and antibiotic sensitivities. ${ }^{12}$ There might be temporal and geographic variations. In addition, it is uncertain whether data obtained from one country or region might be used to draw conclusions for other countries and regions. Therefore, a periodic evaluation of causative organisms and antibiotic sensitivities in a particular geographic region is necessary to ensure that the available treatments are appropriate and effective.

Treatment guidelines for therapy were provided by the EVS. ${ }^{7}$ However, since the spectrum of causative agents might differ among geographic regions, these guidelines might not be universally applicable.

In this study, we examined the spectrum of bacterial isolates that caused endophthalmitis after cataract surgery at our referral centre in Northern Taiwan during the past 6 years to determine whether there are differences from findings reported in other regions, and we analysed the antibiotic susceptibility of bacterial isolates, clinical features, and visual outcomes.

\section{Materials and methods}

The study was approved by the Institutional Review Board of the Tri-service General Hospital. We retrospectively reviewed the medical records of all patients at our centre who were diagnosed with acute bacterial endophthalmitis after cataract surgery, either extracapsular cataract extraction or phacoemulsification, from January 2002 to December 2008.

Patients who had undergone any ocular surgery other than cataract extraction, had any underlying ocular disease other than cataract, presented with endophthalmitis $>6$ weeks after cataract surgery, had traumatic endophthalmitis, or had endogenous endophthalmitis were excluded.

The diagnosis of acute endophthalmitis was made clinically and/or proved by culture. All patients were examined and treated by the same retinal specialist (Dr JT Chen). The treatments generally followed the EVS guidelines. ${ }^{7}$ All patients underwent either intraocular antibiotics injection (IOAI, vancomycin, and amikacin) only or pars plana vitrectomy (PPV) and IOAI. The antibiotics therapy consisted initially of broad spectrum, topical, intravitreal, and systemic antibiotics, which was then tailored according to the culture results, antibiotic susceptibility, and clinical response. The antibiotics commonly used systemically included gentamicin and cephazolin. No systemic steroid was given in any patients. Aqueous and/or vitreous samplings were performed through needle taps for all patients before any intraocular intervention and were subjected to microbiological evaluation. We performed PPV on eyes presenting with light perception vision. Patients with visual acuity (VA) of hand motion or better underwent PPV if any of the following criteria were met: (1) if the patients have any systemic factors, which may compromise immunological status, such as diabetes mellitus and (2) if the clinical signs were toxic and fulminant, such as severe cornea oedema, absence of light reflex, marked intraocular inflammation, and dense non-clearing vitreous opacity. However, some of the patients without these factors also underwent PPV if the surgeon thought it to be in the patient's best interest.

Data were collected on age, gender, type of surgery, percentage of referrals, time to presentation, presenting VA, characteristics of initial presentation (hypopyon, corneal wound suture), complications of initial cataract surgery (posterior capsule rupture, anterior vitrectomy during cataract surgery), initial treatment of endophthalmitis at our retina service, and culture results.

\section{Statistical analysis}

Categorical variables were expressed as count and percentages, and were compared by the Fisher's exact test. Two continuous variables, age and time to presentation, were not normally distributed and were summarized by median and interquartile range, and compared by the Mann-Whitney's test. Simple and multiple linear regression models were performed by their weight coefficients and $95 \%$ confidence interval (CI) of weight coefficients to evaluate important factors for final VA. The variables that had significant impact on final VA in simple linear regression models were stepwise included into the multiple linear regression model. Snellen VA was transformed to the logarithm of the minimum angle of resolution vision (logMAR) in all linear regression models. All statistical analyses were set with a significance level of 0.05 and performed by SPSS 15.0 statistic software (SPSS Inc., Chicago, IL, USA).

\section{Results}

\section{Prevalence and susceptibility for isolates}

The medical records of 59 patients (34 men and 25 women; median age 63.1 years) with acute bacterial 
Table 1 Prevalence of isolates that caused the acute endophthalmitis

\begin{tabular}{lc}
\hline & $\begin{array}{c}\text { Total count } \\
\text { (n=34) }\end{array}$ \\
\hline Gram positive & 15 \\
Staphylococcus aureus & 8 \\
Enterococcus & 4 \\
Streptococcus pneumoniae & 1 \\
Paenibacillus glucanolyticus & 1 \\
Coagulase-negative staphylococci & 1 \\
Gram negative & \\
Pseudomonas aeruginosa & 19 \\
Proteus vulgaris & 13 \\
Stenotrophomonas maltophilia & 2 \\
Moraxella cataralis & 2 \\
\end{tabular}

endophthalmitis after cataract surgery from January 2002 to December 2008 were reviewed. Among these 59 patients, $53(89.8 \%)$ were performed by phacoemulsification, $48(81.3 \%)$ were referred from several local facilities, and $34(57.6 \%)$ had positive cultures. The prevalence of isolates is summarized in Table 1. Among the 34 culture-positive isolates, 19 (55.9\%) were Gram negative and 15 (44.1\%) were Gram positive. The most commonly isolated organism was Pseudomonas aeruginosa $(n=13,38.2 \%)$. Next most common were Staphylococcus aureus $(n=8,23.5 \%)$ and Enterococcus species $(n=4,11.8 \%)$. The results of susceptibility tests for the bacterial isolates are shown in Table 2. Thirty organisms were tested for ciprofloxacin, ceftazidime, cephazolin, and amikacin; 34 were tested for gentamicin and ampicillin; and 18 were tested for vancomycin. Generally, our data showed that the empiric antibiotic regimen used in the EVS was an effective regimen in our geographic region.

\section{Patient characteristics}

Patient baseline characteristics with regard to final VA were summarized in Table 3. Gender, presence of hypopyon, and presenting VA was significantly associated with final VA. Men had significantly better final VA than women $(P=0.01) ; 15(83.3 \%)$ of 18 patients who had final VA better than $5 / 200$ were male. Patients who presented with hypopyon had significantly worse final VA than those without hypopyon $(P=0.007) ; 39$ (95.1\%) of 41 patients who had final VA worse than 5/200 presented with hypopyon. Patients with worse presenting VA had significantly worse final VA than those without $(P<0.001)$.
Table 2 Summary of susceptibility tests for 34 bacterial isolates by Gram negative and Gram positive

\begin{tabular}{|c|c|c|c|}
\hline & $\begin{array}{l}\text { Gram negative } \\
\quad(\mathrm{n}=19)\end{array}$ & $\begin{array}{l}\text { Gram positive } \\
(\mathrm{n}=15)\end{array}$ & $\begin{array}{c}\text { Total } \\
(\mathrm{n}=34)\end{array}$ \\
\hline \multicolumn{4}{|l|}{ Ciprofloxacin ${ }^{\mathrm{a}}$} \\
\hline Susceptible & $18(94.7 \%)$ & $11(100.0 \%)$ & $29(96.7 \%)$ \\
\hline Resistant & $1(5.3 \%)$ & $0(0.0 \%)$ & $1(3.3 \%)$ \\
\hline \multicolumn{4}{|l|}{ Ceftazidime $e^{\mathrm{a}}$} \\
\hline Susceptible & $18(94.7 \%)$ & $11(100.0 \%)$ & $29(96.7 \%)$ \\
\hline Resistant & $1(5.3 \%)$ & $0(0.0 \%)$ & $1(3.3 \%)$ \\
\hline \multicolumn{4}{|l|}{ Cephazolin ${ }^{\mathrm{a}}$} \\
\hline Susceptible & $17(89.5 \%)$ & $11(100.0 \%)$ & $28(93.3 \%)$ \\
\hline Resistant & $2(10.5 \%)$ & $0(0.0 \%)$ & $2(6.7 \%)$ \\
\hline \multicolumn{4}{|l|}{ Amikacin $^{\mathrm{a}}$} \\
\hline Susceptible & $17(89.5 \%)$ & $10(90.9 \%)$ & $27(90.0 \%)$ \\
\hline Resistant & $2(10.5 \%)$ & $1(9.1 \%)$ & $3(10.0 \%)$ \\
\hline \multicolumn{4}{|l|}{ Gentamicin } \\
\hline Susceptible & $13(68.4 \%)$ & $14(93.3 \%)$ & $27(79.4 \%)$ \\
\hline Resistant & $6(31.6 \%)$ & $1(6.7 \%)$ & $7(20.6 \%)$ \\
\hline \multicolumn{4}{|l|}{ Ampicillin } \\
\hline Susceptible & $17(89.5 \%)$ & $10(66.7 \%)$ & $27(79.4 \%)$ \\
\hline Resistant & $2(10.5 \%)$ & $5(33.3 \%)$ & $7(20.6 \%)$ \\
\hline \multicolumn{4}{|l|}{ Vancomycin $^{\mathrm{b}}$} \\
\hline Susceptible & $3(100.0 \%)$ & $15(100.0 \%)$ & $18(100.0 \%)$ \\
\hline Resistant & $0(0.0 \%)$ & $0(0.0 \%)$ & $0(0.0 \%)$ \\
\hline
\end{tabular}

Data are expressed by count and percentage.

${ }^{a}$ Four results were not available in Gram-positive isolates.

bSixteen results were not available in Gram-negative isolates.

\section{Factors affecting final VA}

The results of simple and multiple regression models to identify the important factors that may impact final VA are shown in Table 4 . In the simple linear regression models for final VA, four variables, gender, time to presentation, presence of hypopyon, and presenting VA, were found to have significant impacts on final VA. The four variables except for time to presentation were added stepwise into the multiple linear regression model and it was found that gender, presence of hypopyon, and baseline VA had an independently significant impact on final VA. After adjustment for hypopyon and presenting VA, males had significantly better final VA than females; the difference was $-0.37[95 \% \mathrm{CI}=(-0.69,-0.05)]$ in $\log$ MAR. After adjustment for gender and presenting VA, patients with hypopyon had significantly worse final VA than those without hypopyon; the difference was 0.58 $[95 \% \mathrm{CI}=(0.11,1.04)]$ in logMAR. After adjustment for gender and hypopyon, patients with worse presenting VA had significantly worse final VA than those without; the difference was $0.88[95 \% \mathrm{CI}=(0.49,1.26)]$ in $\log \mathrm{MAR}$. 
Table 3 Summary of baseline patient characteristics by final visual acuity

\begin{tabular}{|c|c|c|c|c|}
\hline & \multirow[b]{2}{*}{ Total $(\mathrm{n}=59)$} & \multicolumn{2}{|c|}{ Final visual acuity } & \multirow[b]{2}{*}{ P-value } \\
\hline & & Better than $5 / 200(\mathrm{n}=18)$ & Worse than $5 / 200(\mathrm{n}=41)$ & \\
\hline Age $($ year $)$ & $63.1(56.1,67.0)$ & $64.1(61.0,66.0)$ & $63.0(56.1,67.0)$ & 0.817 \\
\hline \multicolumn{5}{|l|}{ Gender $^{\mathrm{b}}$} \\
\hline Male & $34(57.6 \%)$ & $15(83.3 \%)$ & $19(46.3 \%)$ & $0.010^{*}$ \\
\hline Female & $25(42.4 \%)$ & $3(16.7 \%)$ & $22(53.7 \%)$ & \\
\hline Time to presentation ${ }^{\mathrm{a}}$ (day) & $2.0(2.0,4.0)$ & $3.0(2.0,4.0)$ & $2.0(2.0,4.0)$ & 0.516 \\
\hline \multicolumn{5}{|l|}{ Presence of hypopyon ${ }^{\mathrm{b}}$} \\
\hline Yes & $51(86.4 \%)$ & $12(66.7 \%)$ & $39(95.1 \%)$ & $0.007^{*}$ \\
\hline No & $8(13.6 \%)$ & $6(33.3 \%)$ & $2(4.9 \%)$ & \\
\hline \multicolumn{5}{|c|}{ Corneal wound suture at initial visit ${ }^{\mathrm{b}}$} \\
\hline Yes & $11(18.6 \%)$ & $2(11.1 \%)$ & $9(22.0 \%)$ & 0.476 \\
\hline No & $48(81.4 \%)$ & $16(88.9 \%)$ & $32(78.0 \%)$ & \\
\hline \multicolumn{5}{|c|}{ Ruptured posterior capsule at initial visit ${ }^{\mathrm{b}}$} \\
\hline No & $56(94.9 \%)$ & $18(100.0 \%)$ & $38(92.7 \%)$ & 0.546 \\
\hline Yes & $3(5.1 \%)$ & $0(0.0 \%)$ & $3(7.3 \%)$ & \\
\hline \multicolumn{5}{|c|}{ Anterior vitrectomy during surgery ${ }^{\mathrm{b}}$} \\
\hline No & $56(94.9 \%)$ & $18(100.0 \%)$ & $38(92.7 \%)$ & 0.546 \\
\hline Yes & $3(5.1 \%)$ & $0(0.0 \%)$ & $3(7.3 \%)$ & \\
\hline \multicolumn{5}{|l|}{$P P V$ as initial treatment ${ }^{\mathrm{b}}$} \\
\hline Yes & $55(93.2 \%)$ & $16(88.9 \%)$ & $39(95.1 \%)$ & 0.578 \\
\hline No & $4(6.8 \%)$ & $2(11.1 \%)$ & $2(4.9 \%)$ & \\
\hline \multicolumn{5}{|l|}{ Baseline visual acuity ${ }^{\mathrm{b}}$} \\
\hline Better than $5 / 200$ & $11(18.6 \%)$ & $6(33.3 \%)$ & $5(12.1 \%)$ & $0.036^{*}$ \\
\hline Worse than $5 / 200$ & $48(81.4 \%)$ & $12(66.6 \%)$ & $36(75 \%)$ & \\
\hline
\end{tabular}

PPV, pars plana vitrectomy.

${ }^{a}$ Data are expressed as median and IQR, and compared by Mann-Whitney's test.

'Data are expressed as count and percentage, and tested by Fisher's exact test.

${ }^{*} P<0.05$ indicated significant difference between groups.

Table 4 Simple and multiple linear regression models for final visual acuity (logMAR)

\begin{tabular}{|c|c|c|c|c|}
\hline & \multicolumn{2}{|c|}{ Simple linear regression } & \multicolumn{2}{|c|}{ Multiple linear regression } \\
\hline & Weight coefficient $(95 \% \mathrm{CI})$ & P-value & Weight coefficient $(95 \% \mathrm{CI})$ & P-value \\
\hline Age (year) & $-0.01(-0.04,0.01)$ & 0.172 & & \\
\hline Gender (male) & $-0.58(-0.96,-0.19)$ & $0.004^{*}$ & $-0.37(-0.69,-0.05)$ & $0.026^{*}$ \\
\hline Time to presentation (days) & $-0.04(-0.08,-0.01)$ & $0.013^{*}$ & & \\
\hline Presence of hypopyon & $0.92(0.39,1.46)$ & $0.001^{*}$ & $0.58(0.11,1.04)$ & $0.016^{*}$ \\
\hline Corneal wound suture at initial visit & $-0.21(-0.77,0.35)$ & 0.449 & & \\
\hline Ruptured posterior capsule at initial visit & $0.65(-0.45,1.75)$ & 0.241 & & \\
\hline PPV as initial treatment & $-0.28(-1.08,0.52)$ & 0.491 & & \\
\hline Baseline visual acuity (logMAR) & $1.04(0.63,1.46)$ & $<0.001^{*}$ & $0.88(0.49,1.26)$ & $<0.001^{*}$ \\
\hline
\end{tabular}

PPV, pars plana vitrectomy.

${ }^{*} P<0.05$ indicated that $95 \% \mathrm{CI}$ of the weight coefficient did not include zero.

\section{Discussion}

We found that in our study of patients in North Taiwan, Gram-negative bacteria, particularly P. aeruginosa, were the causative agents in the majority of postoperative bacterial endophthalmitis cases after cataract surgery. We also found that visual outcome was generally poor and we identified gender, presence of hypopyon, and presenting VA as predictive factors of outcome. 
There have been attempts to identify factors in cataract surgery that are associated with postoperative endophthalmitis. An European study found that the risk of postoperative endophthalmitis after cataract surgery was increased by use of clear corneal incisions and silicone intraocular lens and decreased by use of intracameral cefuroxime at the end of surgery. ${ }^{13}$ An US study found that cataract surgery performed by residents did not increase the risk of postoperative endophthalmitis compared with cataract surgery performed by surgeons with more experience. ${ }^{14}$

The appropriate treatment of patients with postoperative endophthalmitis after cataract surgery should be guided by the results of epidemiological studies. Evidence from studies published over a number of years suggests that a change in the microbiological spectrum of causative organisms has been occurring. According to a study by Recchia et $a l^{12}$ there has been a significant increase in Gram-positive bacteria as the causative organisms. Gram-positive bacteria have been found to be associated with acute postcataract endophthalmitis in $71-93.4 \%$ of cases. Coagulasenegative staphylococcus, $S$. aureus, and S. epidermidis were the most commonly cultured isolates. ${ }^{7-11}$ In contrast, we found that $57.6 \%$ of isolates were Gram negative.

Our finding that the majority of organisms were Gram negative is attributed to geographic variations. Such variations lead to different clinical characteristics. One possible reason for this finding is that we studied referred out-patients who had been treated at local clinics or who treated themselves with drugs purchased at local drug stores. The antibiotic used at our centre and at local clinics at the start of cataract surgery is $10 \%$ providone iodine, which is used to wash the external eye.

Prophylactic antibiotics were not used to irrigate the eye during surgery. Topical antibiotics and a steroid $(0.1 \%$ fluetholone) were prescribed routinely for all patients. The antibiotics commonly used included gentamicin, tobramycin, and cephazolin. The finding that Gramnegative bacteria, and especially P. aeruginosa, were the most commonly isolated pathogens in our study is worthy of discussion in detail, as few reports have focused on infection of $P$. aeruginosa in acute postoperative bacterial endophthalmitis.

Gram-negative bacteria, including $P$. aeruginosa, are responsible for $<30 \%$ of acute postoperative bacterial endophthalmitis cases in European countries. However, it is a serious problem because of the rapidity of infection and virulence often results in poor visual outcome despite prompt antibiotic treatment. Among our Gramnegative isolates, $P$. aeruginosa was the most common organism $(68.4 \%)$. It is a Gram-negative, aerobic, rodshaped bacterium and a highly virulent opportunistic human pathogen. ${ }^{15}$ P. aeruginosa has not been reported as a common cause of postoperative endophthalmitis after cataract surgery earlier, except for two outbreaks in India. ${ }^{16-18}$ Most of the reported cases seem to be related to contaminated intraocular irrigating solution and the route of phacoemulsifier. ${ }^{19}$ In one of the outbreaks in India, polymerase chain reaction results suggested that cause of the outbreak was contaminated air conditioning. ${ }^{18}$ There have been no reported outbreaks of Pseudomonas-related endophthalmitis after cataract surgery in our country. However, a high percentage of the Pseudomonas-related cases were referred from several local facilities $(n=12,92.3 \%)$. It may be reasonable to assume that there is a greater variability in quality control of surgery in primary eye facilities. This might also be a factor in the different spectrum seen in clinical setting-acquired pathogens compared with hospitalacquired pathogens in Taiwan. ${ }^{20}$ However, we have limited data on intraoperative prophylaxis measures (such as antibiotics in irrigating fluid, intracameral antibiotics) or variations in patient profiles. Studies are warranted to document the source of these bacteria and their pathogenesis in this kind of endophthalmitis.

The yield of vitreous cultures in this study was 59.2\%, which is slightly lower than yields reported in Singapore ${ }^{9}$ $(61.8 \%)$, the $\mathrm{EVS}^{7}$ in the United States (69.3), and Sweden $^{8}(75 \%)$. One reason for low culture positivity in our study might be that just needle aspiration was used rather than diagnostic vitrectomy. The small quantity of culture pathogen available in vitreous samples might produce only modest organism growth, making interpretation difficult. However, some studies have shown little difference between mechanized vitreous biopsy and needle tap with respect to microbiologic yield. ${ }^{21}$ Alternatively, differences between our series and others may reflect differences in investigation techniques and interpretation of the reporting of confirmed or equivocal growth. Another possibility is that there might have been some sterile or less virulent cases, which could have led to false-negative culture results.

A change in antibiotic sensitivity has been reported over the past two decades. ${ }^{22}$ Periodic susceptibility testing should be performed to ensure that the antimicrobials used still provide good efficacy in treating bacterial endophthalmitis after surgery. ${ }^{23}$ All EVS patients were treated with intravitreal vancomycin and amikacin, plus subconjunctival vancomycin, ceftazidime, and dexamethasone, and sometimes systemic antibiotics. ${ }^{7}$ We treated all of our patients with PPV or intravitreal vancomycin and amikacin. Concurrently, patients were randomized to receive systemic antibiotics, but not systemic steroids. The use of a combination regimen (vancomycin and amikacin) still provides good coverage for pathogens after cataract surgery in our 
region, although further study regarding the correlation between clinical outcome and in vitro susceptibility might be needed.

In our study, visual outcome after endophthalmitis was generally poor; only $7(11.9 \%)$ of 59 patients have a VA of $>20 / 40$. Better outcomes have been reported in other studies. Wong and Chee ${ }^{9}$ found that $16(51.6 \%)$ of 31 eyes achieved VA of 20/40 or better in some Asian populations. The EVS, ${ }^{7}$ Lalwani et al, ${ }^{24}$ and Kamalarajah et $a l^{25}$ found that almost $50 \%$ of patients achieved $20 / 40$ or better VA in western countries. The poorer visual outcome in our study may be partly explained by the higher percentage of more fulminant and virulent organisms. A study in a Middle Eastern country reported a similar outcome as our study with only $2(10 \%)$ of 20 patients having VA better than $20 / 40 .{ }^{6}$ On the basis of the finding of a different distribution of causative organisms in our study compared with other studies that had better outcomes suggests that the important predictor for poor visual outcome was $P$. aeruginosa-related cases. The visual outcome of a P. aeruginosa outbreak in India was also poor. ${ }^{18}$ The final VA caused by $P$. aeruginosa is generally poor despite prompt treatment with intravitreal antibiotics, which the organism is sensitive to in vitro. In our Pseudomonas cases, there was inevitably extensive anterior segment involvement with corneal exudates and oedema. Only 1 of 13 such cases achieved final VA better than 5/200 and in 2 there was no light perception. The study by Eifrig et $a^{26}$ showed that the posttreated median VA outcome in the postcataract endophthalmitis patients group is no light perception. It is still a challenge to treat postcataract endophthalmitis caused by P. aeruginosa.

Our analysis of prognostic factors showed that a presenting VA of 5/200 or better was associated with good visual outcome of a final VA of 20/40 or better. Better presenting VA means the possibility of earlier disease course, less damage to intraocular tissue, or less virulent organisms and patients might have a greater chance of good visual outcome. The presence of hypopyon might be related to some factors such as fulminant disease course or wound abnormalities. It is reasonable to think that the presence of hypopyon provides worse visual prognosis. Earlier studies have also shown an association between gender and risk of endophthalmitis, although not all..$^{5,9,13,27,28}$ It might be partly explained by the geographic variation.

Our study has limitations. As most of our patients were referred from physicians outside of our medical centre, we could not definitely evaluate the potential impact of factors such as variations in surgical technique, intraoperative antibiotics, presence of preoperative eye conditions of patients, surgical time, or complications during cataract surgery on the prognosis of endophthalmitis. Although our centre is one of the largest retinal services in North Taiwan, the incidence of postoperative endophthalmitis after cataract surgery could not be accurately determined. In addition, the treatment strategy for each case of endophthalmitis was based on the clinical judgment of the treating physician, even though the general guidelines of the EVS were followed. Some patients with VA of hand motion or better may have undergone PPV for dense non-clearing vitreous opacity.

In summary, Gram-negative bacteria, especially $P$. aeruginosa, were the most commonly isolated pathogens in acute postoperative bacterial endophthalmitis-associated cataract surgery in North Taiwan. Pseudomonas species are less known, but virulent microorganisms causing endophthalmitis. In addition, the combination of vancomycin and amikacin is effective as a firstline and reasonable empirical therapeutic regimen in our region. Finally, male, presence of hypopyon, and a presenting VA of 5/200 or better are prognostic factors for better final VA. We believe that several findings in the current series may be useful for comparison with similar series in other geographic areas.

\section{Conflict of interest}

The authors declare no conflict of interest.

\section{Summary}

What was known before

- In most published literature, Gram-positive bacteria, were the most common organisms associated with acute postcataract endophthalmitis.

What this study adds

- Pseudomonas aeruginosa were the most commonly isolated pathogens in acute postoperative bacterial endophthalmitis-associated cataract surgery in North Taiwan.

\section{References}

1 Brain G, Taylor H. Cataract blindness - challenges for 21st century. Bull World Health Org 2001; 79(3): 249-256.

2 West ES, Behrens A, McDonnell PJ, Tielsch JM, Schein OD. The incidence of endophthalmitis after cataract surgery among the US. Medicare population increased between 1994 and 2001. Ophthalmology 2005; 112(8): 1388-1394.

3 Lloyd JC, Braga-Mele R. Incidence of postoperative endophthalmitis in a high-volume cataract surgicentre in Canada. Can J Ophthalmol 2009; 44(3): 288-292.

4 Carrim ZI, Richardson J, Wykes WN. Incidence and visual outcome of acute endophthalmitis after cataract surgery - the experience of an eye department in Scotland. Br J Ophthalmol 2009; 93(6): 721-725.

5 Lalitha P, Rajagopalan J, Prakash K, Ramasamy K, Prajna NV, Srinivasan M. Postcataract endophthalmitis in South India. Incidence and outcome. Ophthalmology 2005; 112(11): 1885-1890. 
6 Al-Mezaine HS, Kangave D, Al-Assiri A, Al-Rajhi AA. Acute-onset nosocomial endophthalmitis after cataract surgery: incidence, clinical features, causative organisms, and visual outcomes. J Cataract Refract Surg 2009; 35(4): 643-649.

7 Endophthalmitis Vitrectomy Study Group. Results of the Endophthalmitis Vitrectomy Study: a randomized trial of immediate vitrectomy and of intravenous antibiotics for the treatment of postoperative bacterial endophthalmitis. Arch Ophthalmol 1995; 113(12): 1479-1496.

8 Montan P, Lundström M, Stenevi U, Thorburn W. Endophthalmitis following cataract surgery in Sweden. The 1999-2001 national prospective survey. Acta Ophthalmol Scand 2005; 83(1): 7-10.

9 Wong TY, Chee SP. The epidemiology of acute endophthalmitis after cataract surgery in an Asian population. Ophthalmology 2004; 111(4): 699-705.

10 Haapala TT, Nelimarkka L, Saari JM, Ahola V, Saari KM. Endophthalmitis following cataract surgery in southwest Finland from 1987 to 2000. Graefes Arch Clin Exp Ophthalmol 2005; 243(10): 1010-1017.

11 Mollan SP, Gao A, Lockwood A, Durrani OM, Butler L. Postcataract endophthalmitis: incidence and microbial isolates in a United Kingdom region from 1996 through 2004. J Cataract Refract Surg 2007; 33(2): 265-268.

12 Recchia FM, Busbee BG, Pearlman RB, Carvalho-Recchia CA, Ho AC. Changing trends in the microbiological aspects of postcataract endophthalmitis. Arch Ophthalmol 2005; 123(3): 341-346.

13 ESCRS Endophthalmitis Study Group. Prophylaxis of postoperative endophthalmitis following cataract surgery: results of the ESCRS multicenter study and identification of risk factors. J Cataract Refract Surg 2007; 33(6): 978-988.

14 Hollander DA, Vagefi MR, Seiff SR, Stewart JM. Bacterial endophthalmitis after resident-performed cataract surgery. Am J Ophthalmol 2006; 141(5): 949-951.

15 Ohl CA, Pollack M. Infections due to pseudomonas species and related organisms. In: Braunwald E, Fauci AS, Kasper DL, Hauser SL (eds). Harrison's Principles of Internal Medicine, 15th ed., McGraw-Hill: New York, 2001, pp 963-970.

16 Anand AR, Therese KL, Madhavan HN. Spectrum of aetiological agents of postoperative endophthalmitis and antibiotics susceptibility of bacterial isolates. Indian J Ophthalmol 2000; 48(2): 123-128.

17 Gupta A, Gupta V, Gupta A, Dogra MR, Pandav SS, Ray P et al. Spectrum and clinical profile of postcataract surgery endophthalmitis in North India. Indian J Ophthalmol 2003; 51: 139-145.
18 Pinna A, Usai D, Sechi LA, Zanetti S, Jesudasan NC, Thomas PA et al. An outbreak of post-cataract surgery endophthalmitis caused by pseudomonas aeruginosa. Ophthalmology 2009; 116(12): 2321-2326.

19 Zaluski S, Clayman HM, Karsenti G, Bourzeix S, Tournemire A, Faliu B et al. Pseudomonas aeruginosa endophthalmitis caused by contamination of the internal fluid pathways of a phacoemulsifier. J Cataract Refract Surg 1999; 25(4): 540-545.

20 Lauderdale TL, Clifford McDonald L, Shiau YR, Chen PC, Wang HY, Lai JF et al. TSAR participating hospitals. The status of antimicrobial resistance in Taiwan among Gram-negative pathogens: the Taiwan surveillance of antimicrobial resistance (TSAR) program, 2000. Diagn Microbiol Infect Dis 2004; 48(3): 211-219.

21 Han DP, Wisniewski SR, Kelsey SF, Doft BH, Barza M, Pavan PR. Microbiologic yields and complication rates of vitreous needle aspiration versus mechanized vitreous biopsy in the Endophthalmitis Vitrectomy Study. Retina 1999; 19(2): 98-102.

22 Chalita MR, Höfling-Lima AL, Paranhos Jr A, Schor P, Belfort Jr R. Shifting trends in in vitro antibiotic susceptibilities for common ocular isolates during a period of 15 years. Am J Ophthalmol 2004; 137(1): 43-51.

23 Eifrig CW, Flynn Jr HW, Scott IU, Newton J. Acute-onset postoperative endophthalmitis: review of incidence and visual outcomes (1995-2001). Ophthalmic Surg Lasers 2002; 33(5): 373-378.

24 Lalwani GA, Flynn Jr HW, Scott IU, Quinn CM, Berrocal AM, Davis JL et al. Acute-onset endophthalmitis after clear corneal cataract surgery (1996-2005). Clinical features, causative organisms, and visual acuity outcomes. Ophthalmology 2008; 115(3): 473-476.

25 Kamalarajah S, Silvestri G, Sharma N, Sharma NK, Cole $\mathrm{MD}, \mathrm{Cran} \mathrm{G}$ et al. Surveillance of endophthalmitis following cataract surgery in the UK. Eye 2004; 18(6): 580-587.

26 Eifrig CW, Scott IU, Flynn Jr HW, Miller D. Endophthalmitis caused by pseudomonas aeruginosa. Ophthalmology 2003; 110(9): 1714-1717.

27 Kamalarajah S, Ling R, Silvestri G, Sharma NK, Cole MD, Cran $G$ et al. Presumed infectious endophthalmitis following cataract surgery in the UK: a case-control study of risk factors. Eye (Lond) 2007; 21(5): 580-586.

28 Kodjikian L, Beby F, Rabilloud M, Bruslea D, Halphen I, Fleury $\mathrm{J}$ et al. Influence of intraocular lens material on the development of acute endophthalmitis after cataract surgery? Eye (Lond) 2008; 22(2): 184-193. 